\title{
A Case of High-Grade Neuroendocrine Carcinoma That Improved with Bevacizumab plus Modified FOLFOX6 as the Fourth-Line Chemotherapy
}

\author{
Satoshi Takeuchi ${ }^{\mathrm{a}}$ Rio Honma ${ }^{\mathrm{a}}$ Jun Taguchi ${ }^{\mathrm{a}}$ \\ Toraji Amano ${ }^{a}$ Yasushi Shimizu $^{a} \quad$ Ichiro Kinoshita $^{a}$ \\ Kanako Kubota ${ }^{b}$ Yoshihiro Matsuno ${ }^{b}$ \\ Hirotoshi Dosaka-Akita ${ }^{a}$
}

aDepartment of Medical Oncology, Hokkaido University Graduate School of Medicine, and 'Department of Surgical Pathology, Hokkaido University Hospital, Sapporo, Japan

\section{Key Words}

High-grade neuroendocrine carcinoma · Small-cell lung cancer · Bevacizumab - Liver origin

\begin{abstract}
High-grade neuroendocrine carcinoma differs from usual neuroendocrine carcinoma, and its prognosis is dismal. In this case report, a case of high-grade neuroendocrine carcinoma that improved with bevacizumab plus modified FOLFOX6 as the fourth-line chemotherapy is presented. A 29-year-old male with a huge liver tumor was diagnosed with high-grade neuroendocrine carcinoma originating from the liver. Multiple liver and bone metastases were found one month after surgery. He was treated with three chemotherapy regimens used for the management of small-cell lung cancer with extensive disease. However, none of them could be maintained because of tumor progression. He was then treated with bevacizumab plus modified FOLFOX6 as the fourth-line regimen. Dramatic tumor shrinkage was obtained, and a partial response was achieved. This case suggests that high-grade neuroendocrine carcinoma can be treated with bevacizumab in combination with cytotoxic chemotherapy.
\end{abstract}




\section{Introduction}

Neuroendocrine carcinoma is classified pathologically into three broad categories based on the grade of differentiation: low grade (well differentiated), intermediate grade (moderately differentiated), and high grade (poorly differentiated) [1]. The high-grade malignancies are aggressive and usually require combined multimodality treatment. When metastatic lesions are present, first-line systemic chemotherapy with a platinumbased regimen that is used in the management of small-cell lung cancer is recommended [2]. A study explored infusional cisplatin and etoposide in 53 patients with metastatic high-grade neuroendocrine carcinoma [3]; the response rate was $42 \%$, and the response duration and median survival were 9 months and 15 months, respectively.

Here, a case with high-grade neuroendocrine carcinoma that improved with bevacizumab plus modified FOLFOX6 (mFOLFOX6) as the fourth-line chemotherapy is presented.

\section{Case Report}

A 29-year-old male with a huge liver tumor was admitted to the Hokkaido University Hospital. The patient had been in good health until two weeks earlier when he felt sudden abdominal pain. On physical examination, an abdominal mass was palpable in the right upper quadrant. A computed tomography (CT) and ${ }^{18} \mathrm{~F}$-fluorothymidine-positron emission tomography (PET) scan revealed a huge liver tumor (over $10 \mathrm{~cm}$ ) and lymph node metastases (fig. 1). There was no evidence of hepatitis B or C virus infection, and he did not have a history of excessive alcohol consumption and smoking. Upper and lower gastrointestinal endoscopy revealed no abnormality. As he had enough visceral function, an extended right lobe hepatectomy was performed. Histopathological examination revealed high-grade neuroendocrine carcinoma originating from the liver (fig. 2). The pathological stage was pT4N1M0, pStage IVA. Despite surgery, multiple liver and bone metastases were found on the follow-up examination one month after surgery. Treatment as for small-cell lung cancer with extensive-stage disease was then chosen. Zoledronic acid and palliative radiotherapy were given for bone metastases. For systemic therapy, he received three chemotherapy regimens used for the management of small-cell lung cancer: two courses of cisplatin plus irinotecan as the first line, two courses of amrubicin as the second line, and one course of topotecan as the third line. However, none of them could be maintained because of aggressive tumor progression.

Usual cytotoxic chemotherapies were thought to be ineffective. However, his Eastern Cooperative Oncology Group (ECOG) performance status (PS) was well maintained (PS 1), and he and his family strongly wished to receive more systemic chemotherapy using other drugs. Therefore, bevacizumab, anti-vascular endothelial growth factor (VEGF) antibody, plus mFOLFOX6 was chosen as his fourthline regimen. The dose and administration schedule were as follows: bevacizumab $5 \mathrm{mg} / \mathrm{kg}$ in $30 \mathrm{~min}$, oxaliplatin $85 \mathrm{mg} / \mathrm{m}^{2}$ in $2 \mathrm{~h}$, L-leucovorin $200 \mathrm{mg} / \mathrm{m}^{2}$ in $2 \mathrm{~h}, 5-\mathrm{FU} 400 \mathrm{mg} / \mathrm{m}^{2}$ in $5 \mathrm{~min}$, and continuous 5 -FU 2,400 mg/m in $46 \mathrm{~h}$. The therapy was given every two weeks. After four cycles of this chemotherapy regimen, the metastases shrank markedly (fig. 3). No adverse events related to bevacizumab were observed. This treatment was continued for eight cycles, and then followed by a regimen without oxaliplatin (i.e. bevacizumab plus simplified LV5FU2 regimen) because of oxaliplatinrelated neurotoxicity. The treatment interval was modified from every two weeks to every three weeks because of thrombocytopenia. Tumor shrinkage continued for over ten months, during which bevacizumab was continued. The patient is still alive at over two years from his first visit, and is continuing bevacizumab in combination with cytotoxic chemotherapy. 


\section{Discussion}

Since neuroendocrine cells are distributed widely throughout the body, neuroendocrine neoplasms can occur in various organs. High-grade neuroendocrine carcinomas can originate for example in the gastrointestinal tract, bladder, cervix or prostate. Cho et al. [4] reviewed prospectively maintained databases from two institutions of patients who underwent hepatic resection for neuroendocrine neoplasms between 1990 and 2006. Only $10 \%$ of 70 patients had high-grade disease. The patient in this case report was diagnosed on histopathological examination as neuroendocrine carcinoma originating from the liver. In addition, the tumor was more aggressive than most neuroendocrine carcinomas (the MIB-1 index was about 70\%), and was classified as highgrade neuroendocrine carcinoma. Systemic surveys revealed no other primary site. This diagnosis is thought to be correct because the patient has shown no evidence of another primary site of neuroendocrine carcinoma over two years from diagnosis.

This case report suggests that high-grade neuroendocrine carcinomas can be treated with bevacizumab in combination with cytotoxic agents. It is plausible that mFOLFOX6 rather than bevacizumab was effective for this patient. However, cisplatin, a platinumcontaining drug similar to oxaliplatin, had already been used in the first-line chemotherapy. 5-FU was used for the first time in this fourth-line regimen, but is not recommended in the guideline for small-cell lung cancer [5]. On the other hand, a phase II study reported the efficacy and safety of bevacizumab plus cisplatin and etoposide in previously untreated patients with extensive-disease small-cell lung cancer [6]. The addition of bevacizumab to cytotoxic chemotherapy improved progression-free survival (PFS) and overall survival (OS) compared with historical controls who had received this chemotherapy regimen without bevacizumab. In this study, PFS and OS were 4.7 and 10.9 months, respectively. Toxicities increased minimally compared with chemotherapy alone in this study. Because bevacizumab was approved only for metastatic colorectal cancer in Japan at the time of first administration of bevacizumab for this patient (July 2009), we had to choose the mFOLFOX6 or FOLFIRI regimen (using irinotecan instead of oxaliplatin) as combination chemotherapy in order to use bevacizumab. Additionally, there are no definite noninvasive biomarkers of anti-VEGF therapy. Current evidence indicates only some predictive value for hypertension, vascular imaging, and VEGF gene polymorphisms as potential biomarkers [7]. To the best of our knowledge, there are no reports about the effectiveness of bevacizumab or mFOLFOX6 in high-grade neuroendocrine carcinoma. In this case report, the addition of bevacizumab seemed to be effective even after the patient was heavily treated with cytotoxic chemotherapy.

Angiogenesis is a fundamental event in tumor growth and metastasis. VEGF is a key regulator of angiogenesis. The addition of bevacizumab, a monoclonal antibody against VEGF, to standard cytotoxic agents is reported to increase overall survival in non-smallcell lung cancer (non-squamous cell carcinoma) and colorectal cancer. The clinical efficacy of bevacizumab in other kinds of cancers still remains to be studied. In preclinical experiments, sustained VEGF inhibition has been shown to achieve and maintain tumor regression [8]. Also in the clinical setting, one observational study suggested that sustained VEGF inhibition could be effective in colorectal cancer [9]. In advanced neuroendocrine carcinoma, targeting therapies seem to be the best real hope for treatment because of insufficiency of conventional cytotoxic chemotherapeutic agents. The rationale behind their investigation is that neuroendocrine carcinomas are highly 
vascular and have been shown to express several angiogenic factors including VEGF [10]. Future clinical studies are required to confirm the efficacy of bevacizumab in the treatment for high-grade neuroendocrine carcinoma.

\section{Disclosure Statement}

We have no conflicts of interest to disclose and no sponsorship or funding arrangements relating to this research.
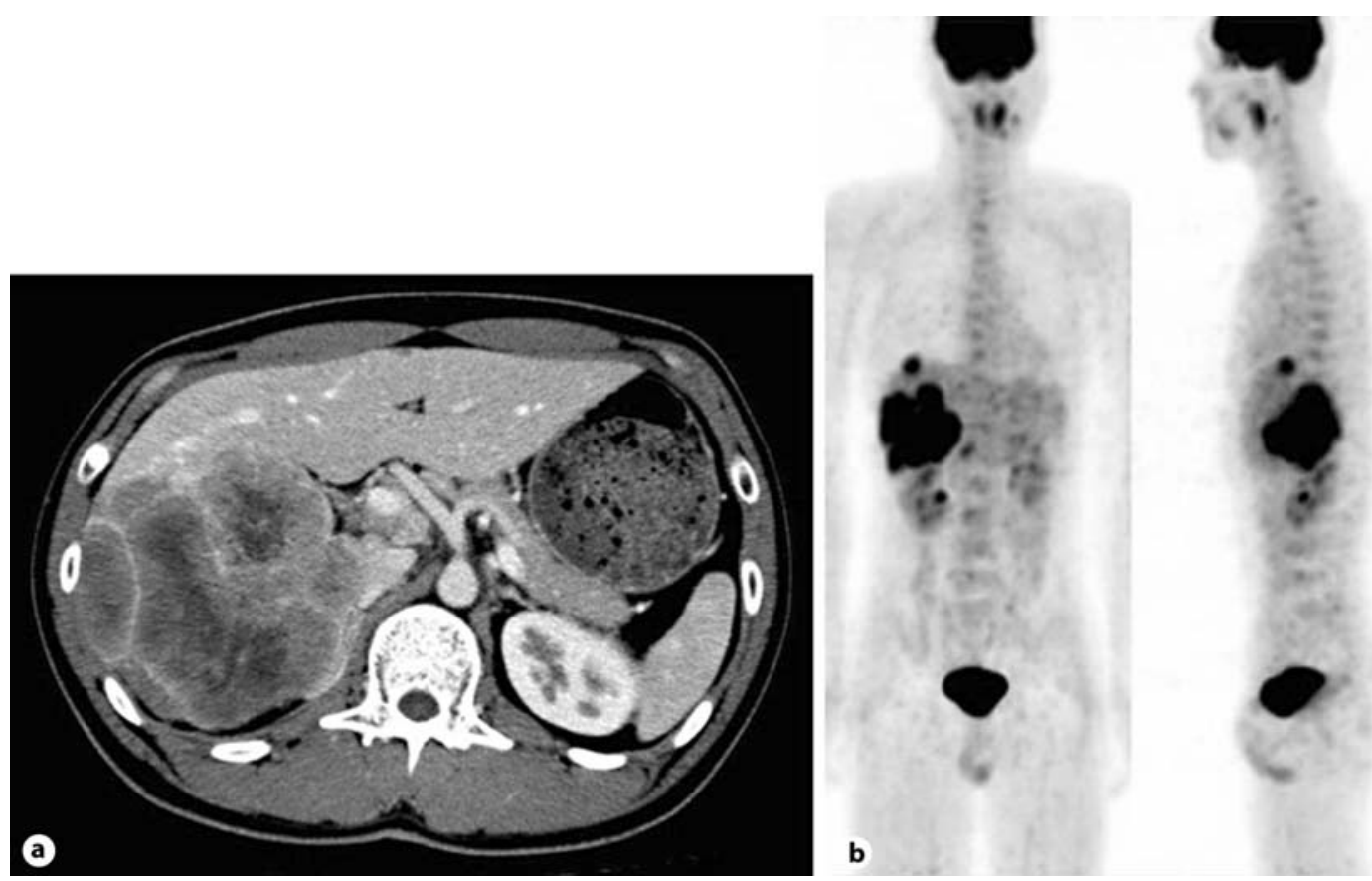

Fig. 1. a CT scan at the first visit. A huge tumor (over $10 \mathrm{~cm}$ ) is seen in the right liver lobe, along with liver metastasis and lymph node swelling. $\mathbf{b}{ }^{18} \mathrm{~F}$-FDG-PET scan at the first visit. No distant metastases were detected. The primary lesion was thought to be in the liver. 

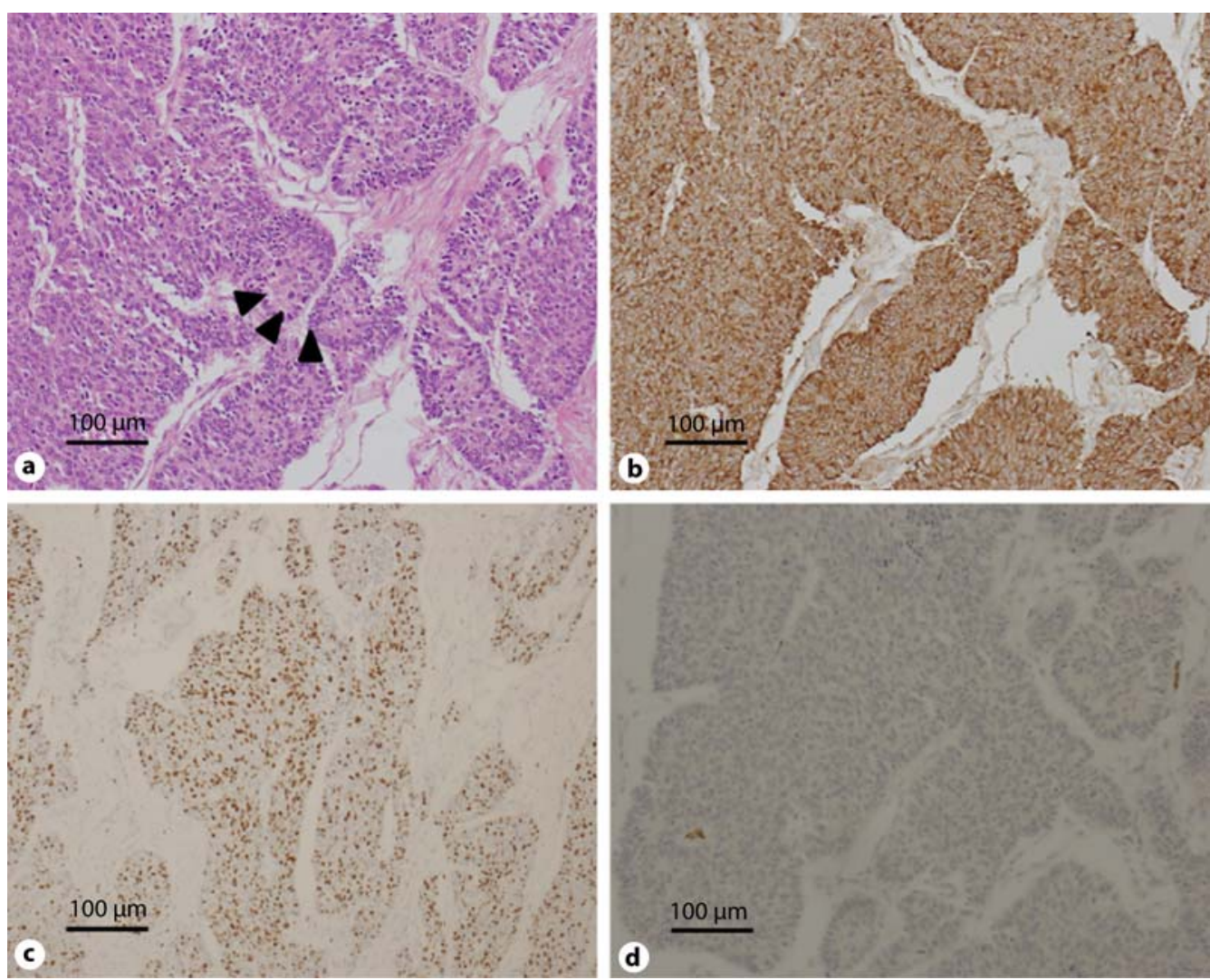

Fig. 2. Histopathological examination reveals high-grade neuroendocrine carcinoma. a Hematoxylin and eosin staining shows tumor cells are characterized by a solid and trabecular architecture. The forming of rosettes are seen (arrowheads). Tumor cells have nuclear pleomorphism and granular cytoplasm. b Chromogranin A immunostaining is seen in almost all carcinoma cells. c The MIB-1 index is about $70 \%$ in the lesion with the highest value. $\mathbf{d}$ TTF-1 immunostaining is negative. 

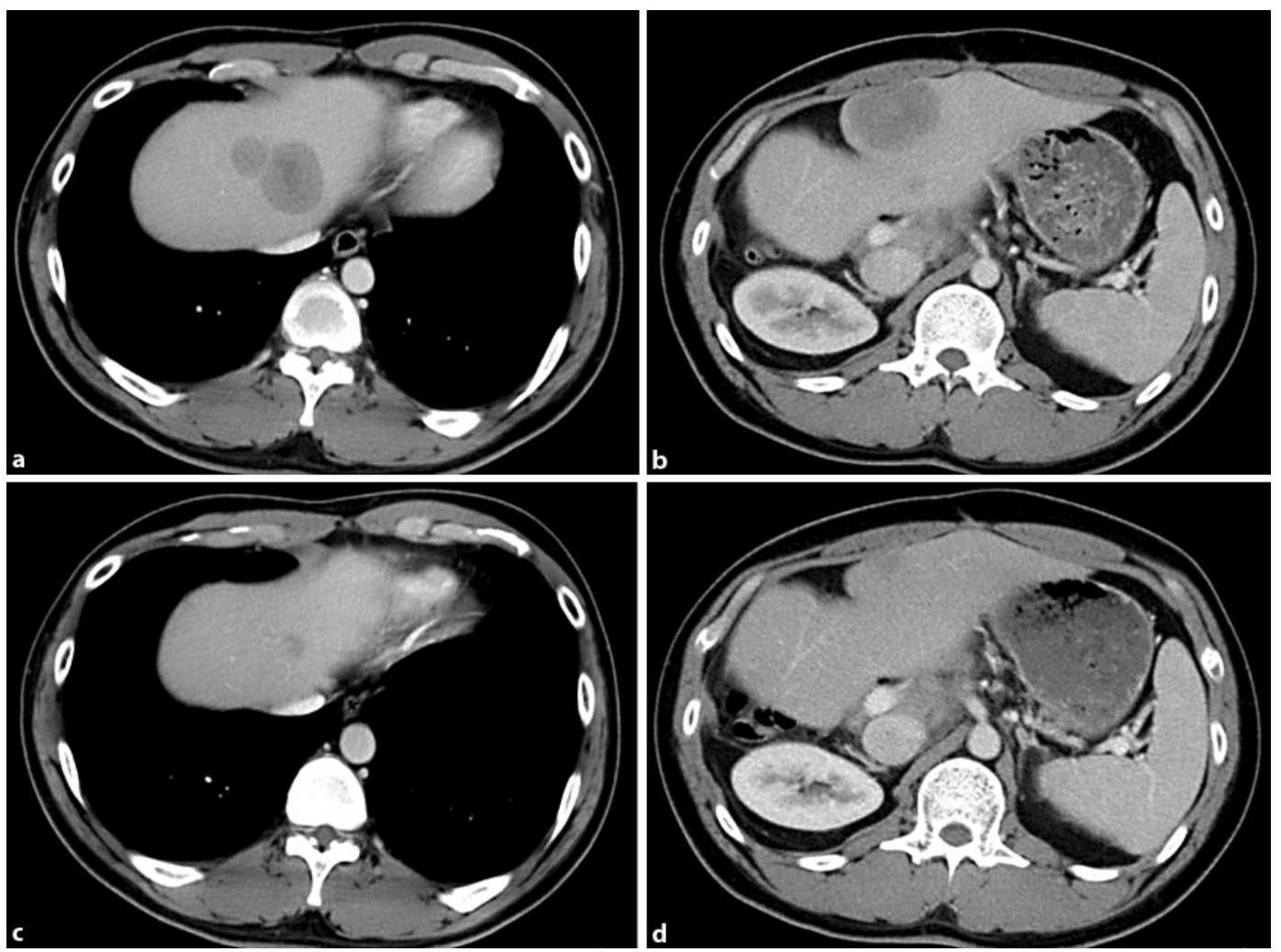

Fig. 3. a, b CT scan prior to chemotherapy with bevacizumab plus mFOLFOX6. Three cytotoxic regimens for small-cell lung cancer with extensive-stage disease have been given by this point. c, d CT scan after four cycles of bevacizumab plus mFOLFOX6. Liver metastases are markedly smaller compared with those before the treatment with this regimen. 


\section{References}

1 NCCN Practice Guidelines in Oncology: Neuroendocrine Tumor. NCCN Practice Guidelines in Oncology, Version 2, 2010

-2 Strosberg JR, Coppola D, Klimstra DS, et al: The NANETS consensus guidelines for the diagnosis and management of poorly differentiated (high-grade) extrapulmonary neuroendocrine carcinomas. Pancreas 2010;39:799-800.

3 Mitry E, Baudin E, Ducreux M, et al: Treatment of poorly differentiated neuroendocrine tumours with etoposide and cisplatin. Br J Cancer 1999;81:1351-1355.

4 Cho CS, Labow DM, Tang L, et al: Histologic grade is correlated with outcome after resection of hepatic neuroendocrine neoplasms. Cancer 2008;113:126-134.

5 NCCN Practice Guidelines in Oncology: Small Cell Lung Cancer. NCCN Clinical Guidelines in Oncology, Version 1, 2011.

6 Horn L, Dahlberg SE, Sandler AB, et al: Phase II study of cisplatin plus etoposide and bevacizumab for previously untreated, extensive-stage small-cell lung cancer: Eastern Cooperative Oncology Group Study E3501. J Clin Oncol 2009;27:6006-6011.

7 Jubb AM, Harris AL: Biomarkers to predict the clinical efficacy of bevacizumab in cancer. Lancet Oncol 2010;11:1172-1183.

8 Klement G, Baruchel S, Rak J, et al: Continuous low-dose therapy with vinblastine and VEGF receptor-2 antibody induces sustained tumor regression without overt toxicity. J Clin Invest 2000;105:R15-R24.

9 Grothey A, Sugrue MM, Purdie DM, et al: Bevacizumab beyond first progression is associated with prolonged overall survival in metastatic colorectal cancer: results from a large observational cohort study (BRiTE). J Clin Oncol 2008;26:5326-5334.

10 Castellano D, Salazar R, Raymond E: Future perspectives on neuroendocrine tumors. Cancer Metastasis Rev 2011;30(suppl 1):35-40. 